\title{
Vi è un'alternativa alla paratiroidectomia nei pazienti con calcolosi renale da iperparatiroidismo primitivo? Tre casi clinici
}

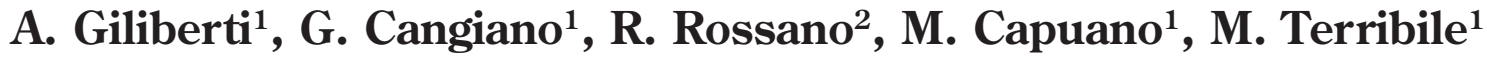 \\ ${ }^{1}$ U.O.C. di Nefrologia e Dialisi e Centro Calcolosi Renale, Ospedale dei Pellegrini, Napoli \\ 2Istituto di Nefrologia Facoltà di Medicina e Chirurgia, Università "Federico II", Napoli
}

\section{Introduzione}

L'iperparatiroidismo (HPT) primitivo è un disordine generalizzato derivante dalla secrezione eccessiva di paratormone $(\mathrm{PTH})$ da parte di una o più ghiandole paratiroidi. Esso è probabilmente la causa più comune di ipercalcemia nella popolazione generale. L'incidenza dell'HPT primitivo aumenta con l'età ed è più elevata nelle donne.

L'esame istologico rivela un adenoma delle paratiroidi in circa il $90 \%$ dei pazienti, sebbene sia talora difficile distinguere un adenoma da una ghiandola normale. Nel $7 \%$ dei casi si evidenzia un'iperplasia e nel 3\% un tumore maligno delle paratiroidi.

L'HPT primitivo determina un'alterazione del metabolismo calcio-fosforo per l'aumentata secrezione di PTH con conseguente ipercalcemia, ipofosfatemia ed eccessivo riassorbimento osseo. $\mathrm{Ne}$ consegue pertanto un aumento del rischio di demineralizzazione ossea e di nefrolitiasi (NL), soprattutto quando l'ipercalciuria è di lunga durata, come si verifica in circa il $10 \%$ dei pazienti affetti.

La chirurgia è il trattamento di scelta dell'HPT primitivo se la diagnosi di localizzazione è certa o molto sospetta, e se sono presenti almeno 4 dei criteri clinici e laboratoristici per paratiroidectomia indicate dalle Linee Guida del NIH, come si evidenzia nella Tabella I (1).

La terapia medica va riservata ai casi a rischio chirurgico o in attesa dell'intervento e ha come scopo la riduzione della calcemia. In questi casi, è comunque necessario uno stretto follow-up, con controllo almeno semestrale di calcemia, calciuria e funzionalità renale.

Da alcuni anni sono disponibili i Calciomimetici, il Cinacalcet (C) in particolare, molecole che agiscono sui recettori del calcio presenti sulla cellula paratiroidea (calcium-sensing receptor), inibendo la secrezione di PTH $(2,3)$. Il C è indicato attualmente nell'HPT secondario ad IRC (4-5), nel carcinoma paratiroideo e recentemente anche nell'HPT primitivo. $\mathrm{Si}$ è infatti osservata, in studi recenti, una riduzione dei livelli di calcemia e PTH nell'HPT primitivo trattato con $30 \mathrm{mg} \times 2$ /die di C. Tali studi hanno permesso di inserire l'HPT primitivo tra le indicazioni di utilizzo del C (6-7).

Abbiamo analizzato i casi clinici di tre pazienti con HPT primitivo trattati con $\mathrm{C}$ in quanto non era stato possibile un approccio chirurgico.

TABELLA I - CRITERI CLINICI E LABORATORISTICI PER PARATIROIDECTOMIA NEL PHPT NIH GUIDELINES 2002 (1)

- Calcolosi renale recidivante

- Fratture e/o Sindrome neuro muscolare

- Calcemia $>1 \mathrm{mg} / \mathrm{dL}$ rispetto ai limiti della norma

- Calciuria $24 \mathrm{~h}>400 \mathrm{mg}$

- Clearance della creatinina $<30 \%$ della norma

- MOC T score <-2.5 DS

- Età < 50anni

*La presenza di almeno 1 di questi criteri può rappresentare una indicazione clinica per la paratiroidectomia 


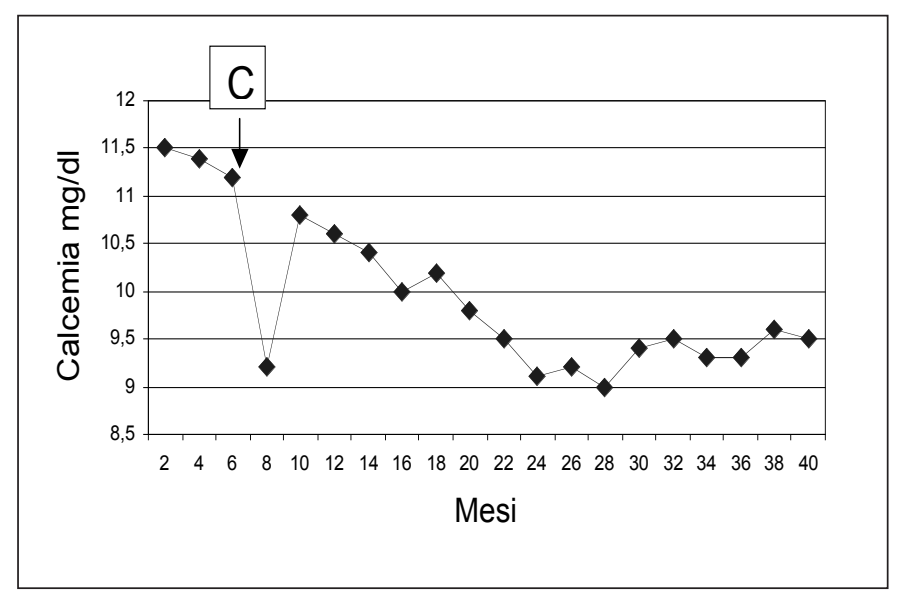

Fig. 1 - Caso 1. Effetti del Cinacalcet sui livelli sierici di Ca durante il periodo di studio.

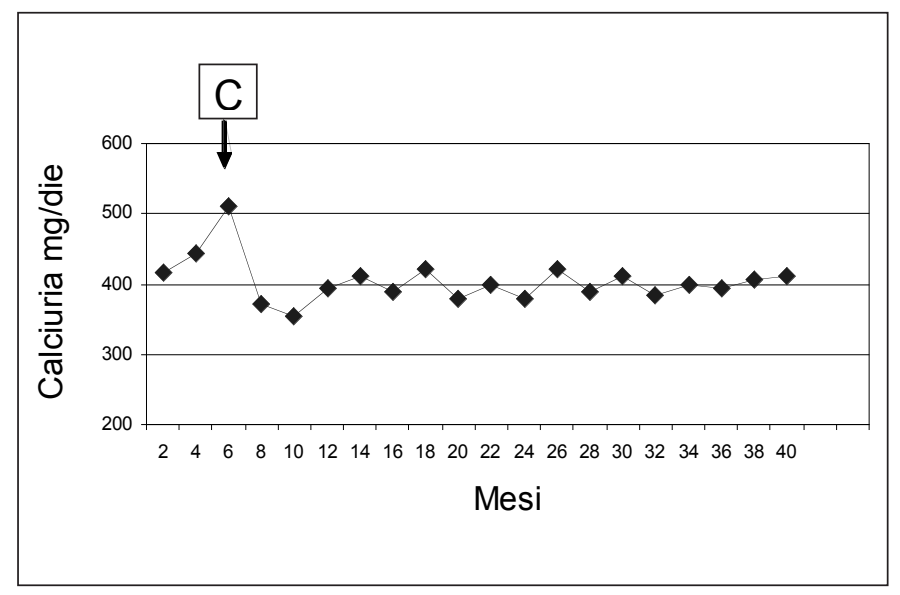

Fig. 3 - Caso 1. Effetti del Cinacalcet sulla escrezione urinaria di Calcio durante il periodo di studio.

\section{CASI CLINICI}

\section{Caso 1}

Paziente S.M. $ð$ di anni 65. Giunge alla nostra osservazione con una storia clinica di NL di vecchia data. Il pz aveva precedentemente eliminato 9 calcoli urinari di cui 5 in seguito a trattamenti di litotripsia extracorporea. L'ecografia renale evidenziava 2 calcoli a dx e 3 calcoli a sn (diametro tra i 6 ed i $9 \mathrm{~mm}$ ).

Lo studio metabolico evidenziava la presenza di HPT primitivo: calcemia 11.8 mg\%, iPTH $130 \mathrm{pg} / \mathrm{mL}$ e calciuria $450 \mathrm{mg} /$ die. Il paziente presentava normofunzione renale con creatininemia $0.9 \mathrm{mg} \%$ e Clearance della creatinina di $70 \mathrm{~mL} / \mathrm{min}$. Inoltre l'esame MOC femore evidenziava osteoporosi con T score $>-2.5$ DS.

E stato quindi iniziato il trattamento con terapia idropinica + citrati + difosfonati.

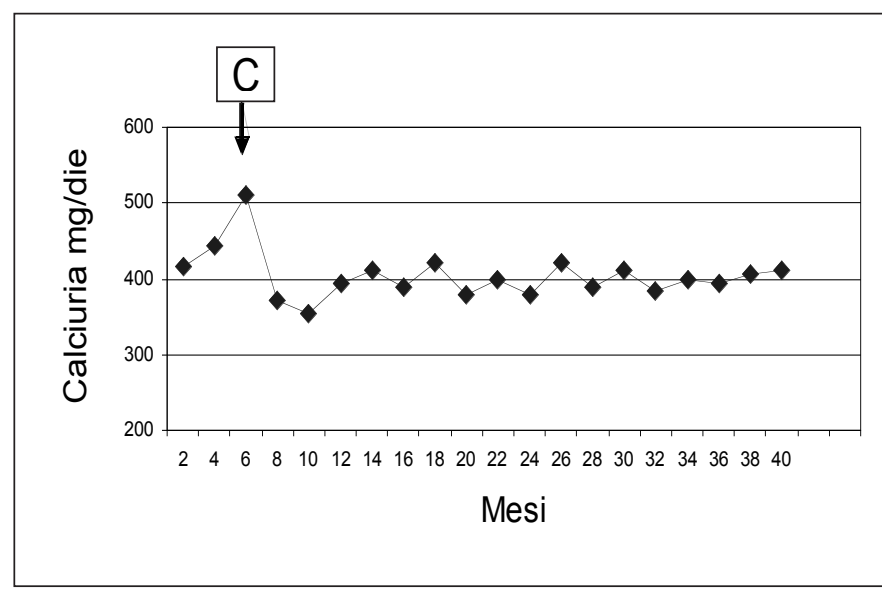

Fig. 2 - Caso 1. Effetti del Cinacalcet sui livelli sierici di PTH durante il periodo di studio.

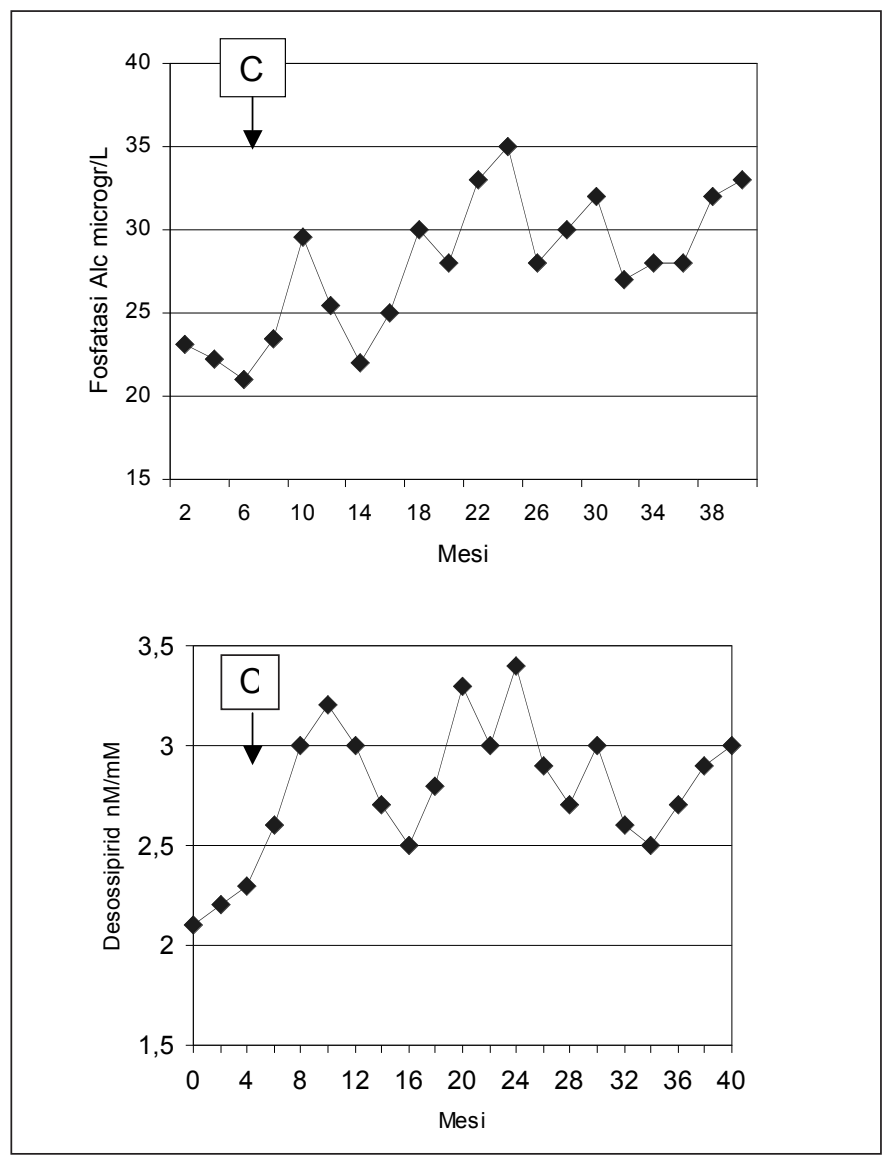

Fig. 4 - Caso 1. Effetti del Cinacalcet sulla fosfatasi alcalina ossea e desossipiridoline urinarie durante il periodo di studio.

Successivamente il pz è stato sottoposto ad esami strumentali: ecografia delle paratiroidi, scintigrafia paratiroidea con sestamibi, TAC cervicotoracica con mdc e RMN cervico toracica. Tali accertamenti non rilevava- 
Fig. 5 - Caso 2. Effetti del Cinacalcet sulla calcemia, PTH e Calciuria durante il periodo di studio. no niente di patologico.

Poiché il pz presentava almeno 4 dei criteri clinici e laboratoristici per paratiroidectomia (Tab. I), è stato sottoposto a visita specialistica di endocrino chirurgia con conseguente proposta di chirurgia esplorativa. Il paziente ha rifiutato l'intervento e pertanto si è continuato il trattamento con terapia idroponica, dieta povera di calcio $(<750 \mathrm{mg} /$ die), citrati e disfosfonati.

Dopo circa 36 mesi, nel maggio 2006, in seguito a visita di controllo presso altra struttura nefrologica, al $\mathrm{pz}$ è stata prescritta terapia con $\mathrm{C}$ alla dose di $30 \mathrm{mg}$ x 2 /die con campionatura gratuita, essendo la prescrizione del C “off label” per l'HPT primitivo.

Dopo circa 4 mesi di terapia il paziente ha praticato nuovamente gli esami ematochimici ed urinari presso il nostro Centro per la Calcolosi. Si evidenziava una riduzione della calcemia (11.4 mg\%) e del iPTH $(100 \mathrm{pg} / \mathrm{mL})$. Il paziente ha poi proseguito la terapia con $\mathrm{C}$ con campionatura gratuita da noi fornita.

L'osservazione del paziente è stata prolungata per un periodo di 40 mesi.
L'andamento di calcemia, iPTH, calciuria, markers di metabolismo minerale osseo quali la fosfatasi alcalina ossea e le desossipiridoline urinarie, è riportato nelle Figure 1- 4.

\section{Caso 2}

Paziente G.A. $\widehat{\jmath}$ di anni 73. Giunge al nostro Centro per la Calcolosi con anamnesi positiva per NL da circa 30 anni con espulsione di 14 calcoli di cui 4 spontaneamente, 4 mediante litotomie e 6 in seguito a litotripsia extracorporea.

Lo studio metabolico da noi effettuato evidenziava HPT primitivo: calcemia $11.0 \mathrm{mg} \%$, iPTH $145 \mathrm{pg} / \mathrm{mL}$ e calciuria di $352 \mathrm{mg} /$ die. La funzionalità renale era nella norma con creatininemia di $1.0 \mathrm{mg} \%$ e Clcr $65 \mathrm{~mL} / \mathrm{min}$. È stata prescritta al paziente terapia idropinica + dieta a moderato contenuto di calcio + citrati.

Le paratiroidi erano esplorate mediante ecografia e scintigrafia con sestamibi senza che si rilevasse alcunché di patologico. 

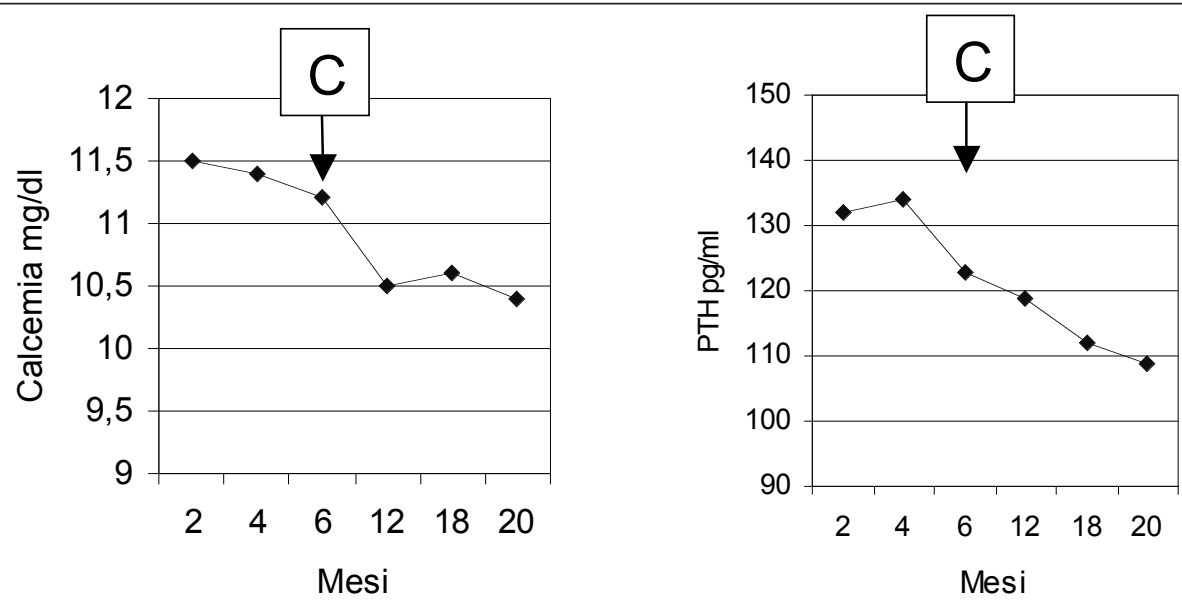

Fig. 6 - Caso 3. Effetti del Cinacalcet sulla calcemia, PTH e Calciuria durante il periodo di studio.

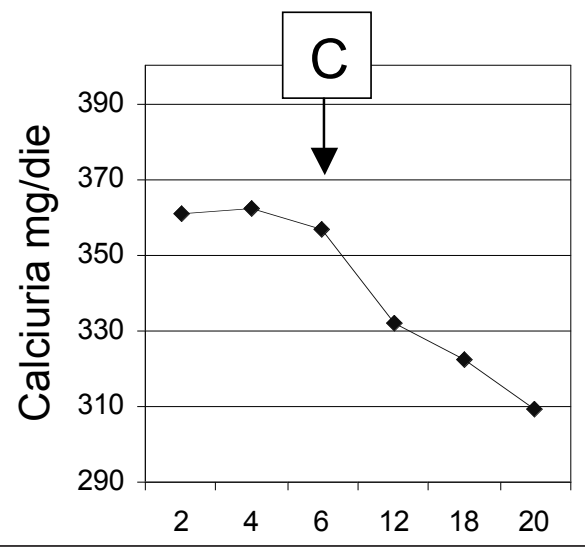

Il paziente ha poi consultato gli endocrinologi che hanno posto erronea diagnosi di HPT secondario ad IRC, in quanto una non corretta raccolta delle urine delle 24 ore ha evidenziato una Clearance della creatinina $<30 \mathrm{~mL} /$ min. Gli è stata prescritta terapia con C $30 \mathrm{mg} /$ die. Il paziente è tornato alla nostra osservazione dopo 6 mesi di terapia con C. Gli esami ematochimici ed urinari hanno rilevato: creat $1.1 \mathrm{mg} \%, \mathrm{Cl}$ creatinina di $62 \mathrm{~mL} / \mathrm{min}$, calcemia $10.5 \mathrm{mg} \%$, iPTH $161 \mathrm{pg} / \mathrm{mL}$ e calciuria $326 \mathrm{mg} /$ die. Ha continuato la terapia prescritta dagli endocrinologi con C alla dose di $30 \mathrm{mg} /$ die. L'osservazione del pz si è protratta per altri 24 mesi ripetendo periodicamente, ogni 6 mesi, gli esami di laboratorio che nel tempo non hanno evidenziato significative variazioni.

Nella Figura 5 è riportato l'andamento di calcemia, iPTH, calciuria.

\section{Caso 3}

Paziente B.P. $q$ di anni 63. È pervenuta alla nostra osservazione con una storia clinica di NL con espulsione spontanea di circa 10 calcoli di calcio ossalato e calcio fosfato.
L'ecografia renale evidenziava alcuni piccoli calcoli caliceali e pelvici bilateralmente (diametro tra i 6 e gli 8 $\mathrm{mm})$. Al rene sn era presente un calcolo caliceale inferiore di $12 \mathrm{~mm}$.

Gli esami di laboratorio evidenziavano HPT primitivo: calcemia $11.5 \mathrm{mg} \%$, iPTH $132 \mathrm{pg} / \mathrm{mL}$ e calciuria $361 \mathrm{mg} /$ die. La funzione renale era nella norma con creatininemia $1.0 \mathrm{mg} \%$ e Clcr $87 \mathrm{~mL} / \mathrm{min}$.

È stato quindi iniziato il trattamento con terapia idropini$\mathrm{ca}+$ dieta a moderato contenuto di calcio + alendronato (70 mg/settimana) + idroclorotiazide.

Successivamente la pz è stata sottoposta ad esami strumentali. L'ecografia delle paratiroidi risultava "nella norma”, la scintigrafia paratiroidea con sestamibi rilevava "nessuna evidenza di tessuto paratiroideo iperfunzionante", la TAC cervicotoracica con mdc e la MOC femore risultavano "nella norma".

Secondo le indicazioni delle Linee Guida (Tab. I), la paziente è stata sottoposta a visita specialistica di endocrino chirurgia che escludeva la possibilità di un intervento di chirurgia esplorativa per l'alto rischio chirurgico in quanto la paziente era affetta da grave obesità.

$\mathrm{Si}$ è proposto allora un trattamento con terapia con $\mathrm{C}$ alla 
dose di $30 \mathrm{mg} \times 2$ /die.

Dopo circa 6 mesi la paziente ha praticato nuovamente gli esami ematochimici che evidenziavano una riduzione della calcemia $(10.8 \mathrm{mg} \%)$ e del PTH $(119 \mathrm{pg} / \mathrm{mL})$. Si è continuata la terapia con $\mathrm{C}$.

L'osservazione della paziente è stata prolungata per un periodo di 20 mesi. Ai controlli ematochimici semestrali non si sono osservate variazioni significative dei parametri del metabolismo osseo.

La Figura 6 mostra l'andamento di calcemia, iPTH, calciuria durante i mesi di osservazione.

\section{DISCUSSIONE}

Nell'HPT il trattamento di scelta è chirurgico se la malattia è sintomatica o progressiva. L'esito dell'intervento dipende dall'effettiva rimozione di tutto il tessuto ipersecernente. Le ghiandole paratiroidi abnormemente funzionanti possono trovarsi in sedi insolite nel collo e nel mediastino e quindi è talvolta necessaria un'esplorazione chirurgica per una corretta localizzazione.

La terapia medica va riservata ai casi a rischio chirurgico per ridurre la calcemia. In questi casi, è comunque necessario uno stretto follow-up, con controllo almeno semestrale di calcemia, calciuria e funzionalità renale (8). Si suggerisce comunque di osservare una dieta a basso contenuto di calcio, di incrementare l'apporto di liquidi e di aumentare l'attività fisica.

I Bifosfonati, farmaci inibitori dell'attività osteoclastica, rappresentano attualmente il farmaco di elezione nel controllo del riassorbimento osseo (9-11). Lattuale di- sponibilità di calciomimetici quali il $\mathrm{C}$ apre prospettive molto più interessanti per la terapia medica.

Il C, indicato inizialmente nel carcinoma paratiroideo e nell'HPT secondario a IRC $(4,5,8)$ è attualmente prescrivibile anche nell'HPT primitivo.

I nostri casi clinici hanno confermato l'efficacia dei calciomimetici ma ad una dose non inferiore ai $30 \mathrm{mg} \mathrm{x}$ $2 /$ die. Infatti, nel Caso clinico 2 , in cui il paziente era sottoposto a terapia con C alla dose di $30 \mathrm{mg} /$ die, non si osserva alcuna riduzione dei parametri ematici e urinari studiati.

In conclusione nei casi in cui siano presenti uno o più noduli paratiroidei, l'intervento chirurgico rappresenta la terapia di elezione e non si possono ipotizzare alternative.

Se invece l'approccio chirurgico appare indaginoso, per l'assenza di evidenti paratiroidi iperfunzionanti, o perché le condizioni cliniche del paziente non permettono di affrontare un intervento chirurgico, la terapia medica con $\mathrm{C}$ può rappresentare un'utile alternativa.

Effetti collaterali di rilievo non sono stati peraltro evidenziati alle dosi comunemente utilizzate di $\mathrm{C}$. Vi è però da segnalare che il costo del farmaco è elevato.

\section{Indirizzo degli Autori:}

Maurizio Terribile, MD

U.O.C. di Nefrologia e Dialisi e Centro Calcolosi Renale Ospedale dei Pellegrini

Via Portamedina alla Pignasecca, 41

80134 Napoli, Italy

maurizio.terribile@fastwebnet.it

\section{Bibliografia}

1. Bilezikian JP, Potts JT, Fuleihan G, et al. Summary statement from a workshop on asymptomatic primary hyperparathyroidism: a perspective for the $21^{\text {st }}$ century. J Clin Endocrinol Metab 2002; 87: 5353-61.

2. Nemeth EF, Steffey ME, Hammerland LG, et al. Calcicimetics with potent and selective activity on the parathyroid calcium receptor. Proc Natl Acad Sci USA 1998; 95: 4040-5.

3. Kawata T, Imanishi Y, Kobayashi K, et al. Direct in vitro evidence of the suppressive effect of cinacalcet HCL on parathyroid hormone secretion in human parathyroid cells with pathologically reduced calciumsensing receptor levels. J Bone Miner Metab 2006; 24: 300-06.

4. Block GA, Martin KJ, de Francisco AL, Turner SA, Avram MM, Suranyi MG, et al. N Engl J Med 2004; 350(15): 1516-25.

5. Lindberg JS, Culleton B, Wong G, Borah MF, Clark $\mathrm{RV}$, Shapiro $\mathrm{WB}$, et al. Cinacalcet $\mathrm{HCl}$, an oral calcimimetic agent for the treatment of secondary hyperpar- 
athyroidism in hemodialysis and peritoneal dialysis: a randomized, double-blind, multicenter study._J Am Soc Nephrol 2005; 16(3): 800-7.

6. Sajid-Crockett S, Singer FR, Hershman JM. Cinacalcet for the treatment of primary hyperparathyroidism. Metabolism 2008; 57 (4): 517-21.

7. Iglesias P, Ais G, González A, Tajada P, García Arévalo C, Fernández Pardo E, Díez JJ. Acute and one-year effects of cinacalcet in patients with persistent primary hyperparathyroidism after unsuccessful parathyroidectomy. Am J Med Sci 2008; 335(2): 111-4.

8. Vestergaard P. Current pharmacological option for the management of primary hyperparathyroidism. Drugs 2006: 66: 2189-211.
9. Selby PL, Peacock M. Ethinyl estradiol and norethindrone in the treatment of primary hyperparathyroidism in postmenopausal womem. N Engl J Med 1996; 314: 1481-5.

10. Chow CC, Chan WB, Li JK, et al. Oral alendronate increases bone mineral density in postmenopausal women with primary hyperparathyroidism. J Clin Endocrinol Metab 2003; 88: 581-7.

11. Orr-Walker BJ, Evans MC, Clearwater JM, Horne A, Grey AB, Reid IR. Effects of hormone replacement therapy on bone mineral density in postmenopausal women with primary hyperparathyroidism: four-year follow-up and comparison with healthy postmenopausal women. Arch Intern Med 2000; 160(14): 2161-6. 